OPEN ACCESS

Edited by:

José Roberto Mineo,

Federal University of Uberlândia, Brazil

Reviewed by:

Dolores Correa

Instituto Nacional de Pediatria, Mexico

Stephen James Jordan,

University of Alabama at Birmingham,

United States

*Correspondence:

Zhiqiang Liu

bydoctorlzq@sina.com

Xuemei Hu

xue-mei-hu@163.com

tThese authors have contributed equally to this work.

Specialty section: This article was submitted to

Microbial Immunology, a section of the journa

Frontiers in Microbiology

Received: 14 December 2017 Accepted: 14 March 2018

Published: 28 March 2018

Citation: Zhan S, Zheng J, Zhang H, Zhao $M$, Liu X, Jiang Y, Yang C, Ren L, Liu Z and Hu $X$ (2018) LILRB4 Decrease on uDCs Exacerbate Abnormal Pregnancy Outcomes Following Toxoplasma gondii Infection.

Front. Microbiol. 9:588. doi: 10.3389/fmicb.2018.00588

\section{LILRB4 Decrease on uDCs Exacerbate Abnormal Pregnancy Outcomes Following Toxoplasma gondii Infection}

\author{
Shaowei Zhan ${ }^{1 t}$, Jing Zheng ${ }^{2 t}$, Haixia Zhang ${ }^{3 t}$, Mingdong Zhao ${ }^{4}$, Xianbing Liu ${ }^{3}$, \\ Yuzhu Jiang ${ }^{3}$, Chunyan Yang ${ }^{3}$, Liqin Ren ${ }^{3}$, Zhiqiang Liu' ${ }^{1 *}$ and Xuemei Hü ${ }^{3 *}$ \\ 1 Department of Gynecology and Obstetrics, Binzhou Affiliated Hospital of Binzhou Medical University, Binzhou, China, \\ 2 Department of Gynecology and Obstetrics, Yantai Traditional Chinese Medicine Hospital, Yantai, China, \\ ${ }^{3}$ Department of Immunology, Department of Medicine \& Pharmacy Research Center, Binzhou Medical University, Yantai, \\ China, ${ }^{4}$ Department of Radiology, Binzhou Affiliated Hospital of Binzhou Medical University, Binzhou, China
}

Toxoplasma gondii (T. gondii) infection in early pregnancy can result in miscarriage, dead fetus, and other abnormalities. The LILRB4 is a central inhibitory receptor in uterine dendritic cells (UDCs) that plays essential immune-regulatory roles at the maternal-fetal interface. In this study, T. gondii-infected human primary uDCs and T. gondii-infected LILRB4 $^{-/-}$pregnant mice were utilized. The immune mechanisms underlying the role of LILRB4 on uDCs were explored in the development of abnormal pregnancy outcomes following T. gondii infection in vitro and in vivo. Our results showed that the expression levels of LILRB4 on uDCs from normal pregnant mice were obviously higher than nonpregnant mice, and peaked in mid-gestation. The LILRB4 expression on uDC subsets, especially tolerogenic subsets, from mid-gestation was obviously down-regulated after T. gondii infection and LILRB4 decrease could further regulate the expression of functional molecules (CD80, CD86, and HLA-DR or MHC II) on uDCs, contributing to abnormal pregnancy outcomes. Our results will shed light on the molecular immune mechanisms of UDCs in abnormal pregnancy outcomes by T. gondii infection.

Keywords: LILRB4, dendritic cells, co-stimulatory molecules, abnormal pregnancy outcomes, Toxoplasma gondii

\section{INTRODUCTION}

Toxoplasma gondii (T. gondii) is an obligate intracellular parasite capable of infecting a wide range of mammalian hosts including humans. Infection by $T$. gondii during pregnancy can cause severe sequelae, such as spontaneous abortion, stillbirth, low birth weight, and significant birth defects for surviving neonates (Robbins et al., 2012). Although several immune mechanisms have been postulated (Xu et al., 2013; Zhao et al., 2013; Liu Y. et al., 2014), the detailed mechanisms underlying adverse pregnancy outcomes following $T$. gondii infections need to be further explored. The microenvironment at the maternal-fetal interface plays an important role in maintaining normal pregnancy (Hunt and Robertson, 1996). Multiple immune cells and cytokines at the maternal-fetal interface participate in protecting the semi-allogeneic embryo from maternal attack and promote immune tolerance during pregnancy (Guleria and Sayegh, 2007). Among these immunocompetent cells at the maternal-fetal interface, antigen-presenting cells (APCs) are 
regarded as important participants in immune regulation during pregnancy (Della Bella et al., 2011).

Dendritic cells are essential for the initiation of primary immune responses and have been reported to induce immunological tolerance and to regulate cell-mediated immune responses (Langenkamp et al., 2000). Uterine dendritic cells (uDCs) are scattered throughout the decidualized endometrium throughout gestation and play vital immune-regulatory role at the maternal fetal interface (Laskarin et al., 2007). In normal human early pregnancy, uDCs include BDCA- $1^{+} \mathrm{CD} 19^{-} \mathrm{CD} 14^{-}$ myeloid DC type 1 (MDC1), BDCA- $3^{+} \mathrm{CD} 14^{-}$myeloid DC type 2 (MDC2), and BDCA-2 ${ }^{+} \mathrm{CD}_{123}{ }^{+}$plasmacytoid DC (PDC) subsets (Ban et al., 2008). MDCs have been reported to induce certain forms of immunity responsible for the maintenance of a normal pregnancy (Gardner and Moffett, 2003). Mice uDCs have been classified into two distinct subsets, CD $11 c^{+} \mathrm{CD} 8 \alpha^{-}$

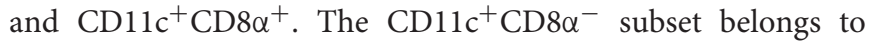
the myeloid lineage and comprises the vast majority of uDCs, mainly exhibiting an immature phenotype and contributing to the induction of maternal-fetal immune tolerance (Blois et al., 2004).

In the periphery, tolerogenic status for DCs is characterized by a low level of the co-stimulatory molecules CD80 and CD86 and high expression of the inhibitory receptors LILRB4 (also called ILT3, gp49B, CD85k) (Adorini et al., 2004). The inhibitory receptor LILRB4, which is mainly expressed on professional APCs, belongs to immunoglobulin superfamily members and contains an immune-receptor tyrosine-based inhibitory motif (ITIM) in their intracellular domains to transduct negative signals (Cella et al., 1997; Kim-Schulze et al., 2006). LILRB4-expressing APC plays prominent roles in controlling inflammation by inhibiting the expression of costimulatory molecules (Chang et al., 2009; Vlad et al., 2010). Further evidence indicated that over-expression of LILRB4 can inhibit the transcription of NF- $\mathrm{B}$-dependent genes that encode co-stimulatory molecules (CD80, CD86) in DCs (Cella et al., 1997). Functional studies have suggested that LILRB4 neutralization can enhance antigen presentation (Regnault et al., 1999; Kasai et al., 2008). At the fetal-maternal interface, in vivo studies showed that LILRB4 mRNA has been detected in murine uterine endometrium during early pregnancy (Matsumoto et al., 1997), and LILRB4 protein expression was detected on immature uDCs of human decidual tissue using flow cytomety (Ban et al., 2008).

Our previous study showed that uDCs contribute to abnormal pregnancy outcomes caused by $T$. gondii infection in early pregnancy (Liu X. et al., 2014). Most importantly, our recent study has reported that LILRB4 on decidual macrophage was involved in the development of abnormal pregnancy outcomes during T. gondii infection (Li et al., 2017). Whether LILRB4 on uDCs also contributed to abnormal pregnancy outcomes after $T$. gondii infection remains unclear, and the associated mechanisms are also unknown. Hence, in the present study, T. gondii-infected human uDCs and T. gondii-infected LILRB $4^{-/}$- pregnant mice were used to explore the mechanisms related to LILRB4 on uDCs that lead to abnormal pregnancy outcomes.

\section{MATERIALS AND METHODS}

\section{Maintenance of T. gondii Tachyzoites (RH Strain)}

The T. gondii tachyzoites were cultured in HEp-2 cells in Minimum Essential Media (MEM) (Hyclone, United States), 5\% fetal bovine serum (FBS; Gibco, United States), and $100 \mathrm{IU} / \mathrm{ml}$ penicillin/streptomycin (Sigma-Aldrich, United States). After culture, tachyzoites were centrifuged at $1500 \mathrm{rpm}(433 \times g)$ for $10 \mathrm{~min}$, and purified tachyzoites were resuspended in MEM and counted using a Neubauer chamber. The experiment was carried out in BSL-2 laboratories. All the liquids, consumables and labwares contaminated with the parasites were collected and steeped immediately in disinfectant, and sterilized by highpressure sterilizer. The mice carcasses were collected in ice locker and transported out by the professionals of public health agencies.

\section{Human Abortion Sample Collection}

Abortion samples of the first-trimester decidua (6-12-week gestation) from elective termination procedures were obtained from Yantai Affiliated Hospital of the Binzhou Medical University. All patients signed an informed consent form before enrollment, and sample collection for this study was approved by the ethics Committee of Binzhou Medical University. The decidual tissues were rinsed in sterile saline solution and rapidly transferred to ice-cold Roswell Park Memorial Institute medium (RPMI). All samples were disposed as soon as possible. Human sample collection procedures for this study were approved by the Binzhou Medical University Ethics Committee, and all patients provided written informed consent for the collection of samples and subsequent analysis.

\section{Human Decidual Cell Preparation and Flow Cytometry Analysis}

Decidual tissues were washed 4-5 times with RPMI medium. Pieces of decidual tissue were minced using the gentle MACS $^{\mathrm{TM}}$ Dissociator (Mitenyi-Biotec, Germany) according to the manufacturer's instructions, and then digested in $0.1 \%$ collagenase type IV and $25 \mathrm{IU} / \mathrm{ml}$ DNase I (both from SigmaAldrich, St. Louis, MO, United States) in RPMI for $45 \mathrm{~min}$ at $37^{\circ} \mathrm{C}$ with gentle rotation. Single cell suspension was filtered twice with a $75 \mu \mathrm{m}$ pore size nylon mesh (Miltenyi Biotec $\mathrm{GmbH}$, Bergisch Gladbach, Germany) and centrifuged at $2000 \mathrm{rpm}(771 \times g)$ for $10 \mathrm{~min}$ at room temperature. The mononuclear cells were then isolated by density gradient separation over a standard FicollHypaque Lymphoprep (1.077, Haoyang Biological Manufacture, Co., Tianjin, China). Decidual mononuclear cells were collected from the interface and were washed twice in cold phosphate buffer solution (PBS). Then the cells were incubated for $12 \mathrm{~h}$ in RPMI supplemented with $10 \%$ FBS, $100 \mathrm{IU} / \mathrm{ml}$ penicillin, and $100 \mathrm{IU} / \mathrm{ml}$ streptomycin in a humidified incubator at $37^{\circ} \mathrm{C}$ with $5 \% \mathrm{CO}_{2}$. After $12 \mathrm{~h}$ of culture, the cells for the infected group were co-cultured with $T$. gondii tachyzoites at a ratio of 1:2 for $12 \mathrm{~h}$ in 6-well culture plates. The LILRB4neutralized infected group was infected at the same condition 
in the presence of anti-IILRB4 neutralizing antibodies (mAbs) (10 $\mu \mathrm{g} / \mathrm{mL}$, eBioscience, United States). The uninfected group was considered as control. Cells were incubated for $12 \mathrm{~h}$ in the same condition as described above. The mononuclear cells were collected and stained with fluorophore-conjugated mAbs: FITCconjugated anti-CD1c (BDCA-1), FITC-conjugated anti-CD303 (BDCA-2), FITC-conjugated anti-CD141 (BDCA-3) (all from Miltenyi Biotec GmbH, Bergisch Gladbach, Germany), PerCPCy5.5-conjugated anti-CD14, CD19, CD123, PE-conjugated antiHLA-DR, PE-conjugated anti-CD80, PE-conjugated anti-CD86 (all from BD Pharmingen, United States), and APC-conjugated anti-LILRB4 (eBioscience, United States). The mAbs were added according to the manufacturer's protocol. After incubation, the cells were washed twice with PBS and resuspended in $600 \mu \mathrm{l}$ of PBS. The cells were detected using BD FACSAria flow cytometry (Becton Dickinson, United States) and the data were analyzed using BD FACSDiva 7.0 software (Becton Dickinson).

\section{Animal Models}

Wild type (WT) female mice (Beijing Weitong Lihua Experimental Animal Technical, Co., Ltd.) and LILRB4 ${ }^{-/-}$ female mice (Experimental Animal Division RIKEN BioResource Center, Japan) at 6- to 8-week-old were mated with the corresponding 8 - to 10 -week-old male mice overnight at a ratio of 2:1 and were checked for vaginal plugs the next morning. Females with a vaginal plug were segregated and designated as gestational day 0 ( Gd 0$)$. The infected group were inoculated intraperitoneally (i.p.) with 400 tachyzoites in $200 \mu$ l sterile PBS on Gd 8. The uninfected groups were inoculated with equivalent PBS at the same time. The protocol of animal experiment was approved by the Committee on the Ethics of Animal Experiments of the Binzhou Medical University. All procedures were performed under sodium pentobarbital anesthesia, and all efforts were made to minimize suffering of the animals.

\section{Mice Mononuclear Cell Preparation and Flow Cytometry}

Mononuclear cells from mouse uterine and placenta were prepared as previously described (Liu X. et al., 2014). Briefly, mice were sacrificed on Gd 14. The uterus and placenta were excised with surgical cuts. The pregnancy outcome was reflected by the total number of fetuses, fetal size, stillbirth, absorptive fetus, and hemorrhagic appearance. The uterus and placentas were washed with sterile PBS, minced into small pieces. Dispersed cells were collected by filtration through a $48 \mu \mathrm{m}$ pore size stainless steel mesh. Thereafter, the mononuclear cells were obtained by density-gradient centrifugation and washed twice in cold PBS. The following fluorochrome-conjugated, mousespecific mAbs were used in the assays: FITC-conjugated antiCD11c (BD Biosciences, United States), Percp-cy5.5-conjugated anti-CD8a mAb (BD Biosciences, United States), PE-conjugated anti-CD80 (BD Biosciences, United States), PE-conjugated

A
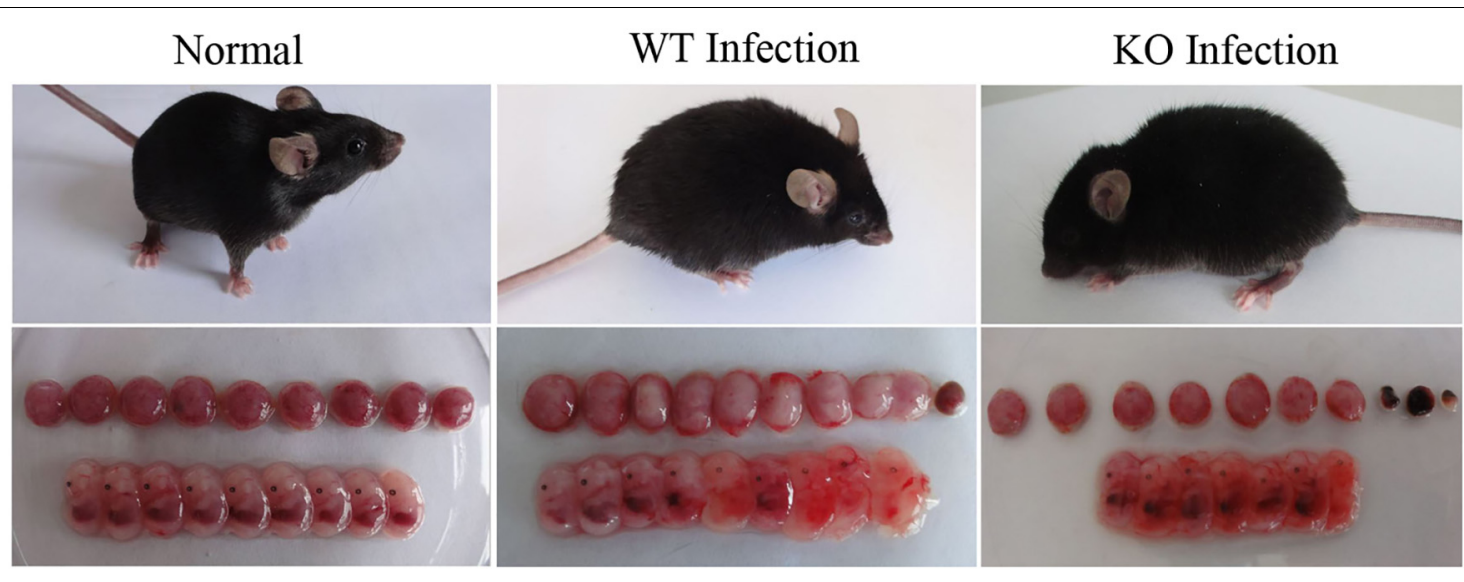

C
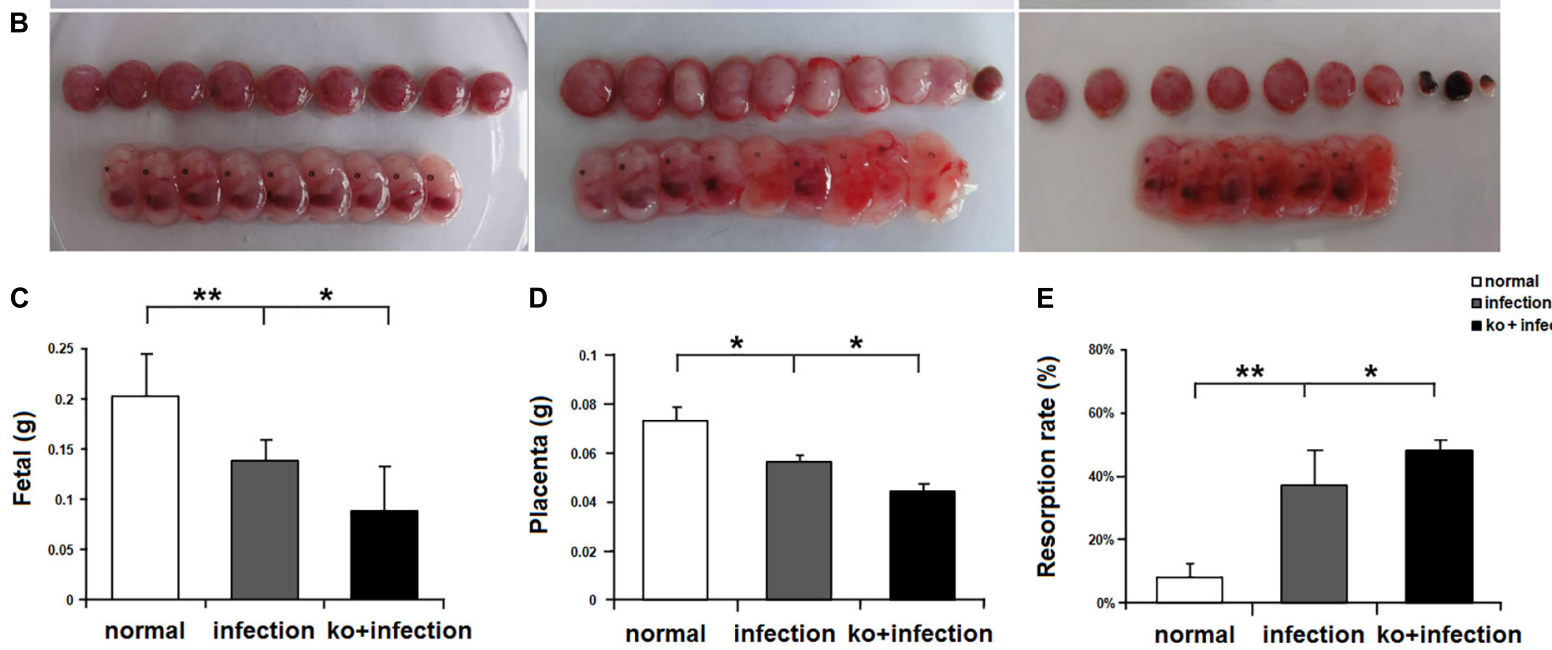

FIGURE 1 | The impact of LILRB4 on abnormal pregnancy outcomes caused by Toxoplasma gondii infection. (A) The pregnant mice and (B) their fetuses from normal wild type (WT) pregnant, T. gondii infected WT pregnant mice, and T. gondii infected LILRB4 ${ }^{-1}$ - pregnant mice. (C) The weight of fetuses, (D) the weight of placentas, and $\mathbf{E}$ ) the resorption rate from the three groups. Data are shown as means \pm SD for 10 pregnant mice and differences were identified using unpaired $t$-tests $\left({ }^{*} p<0.05,{ }^{* *} p<0.01\right)$. 
anti-CD86 (BD Biosciences, United States), PE-conjugated anti-I-Ad/I-Ed (MHC II) (BD Biosciences, United States), and PE-conjugated anti-LILRB4 (Biolegend, United States). Cells were incubated for $30 \mathrm{~min}$ at $4^{\circ} \mathrm{C}$ in the dark according to the manufacturer's instructions. Subsequently, the cells were washed with cold PBS and analyzed using BD FACSAria flow cytometry and BD FACSDiva 7.0 software (Becton Dickinson, United States).

\section{Statistical Analysis}

Data were presented as the mean \pm SEM. Statistical analysis was performed using the SPSS statistics software package (SPSS 17.0; SPSS, Inc., Chicago, IL, United States). Unpaired $t$-tests were used after verifying that the data of the groups had a normal distribution by SAS to compare two independent groups. $p<0.05$ was regarded as significant and $p<0.01$ was considered as very significant.

\section{RESULTS}

\section{The Abnormal Pregnancy Outcomes Were Related to LILRB4 in T. gondii Infected Mice}

Toxoplasma gondii infection during early pregnancy can cause abnormal pregnancy outcomes. In order to explore the effects of LILRB4 on the adverse pregnancy outcome caused by T. gondii, we observed the pregnancy outcomes of $T$. gondii infected LILRB4 $4^{-/}$mice. The results were in accordance with our previous studies (Li et al., 2017). In both the WT and LILRB4 ${ }^{-/-}$infected pregnant group, the pregnant mice were flagging, shambling, and had erected fur, whereas the control mice were nimble, restive, and had smooth pelage (Figure 1A). Compared with WT infection groups, most fetuses from LILRB4 ${ }^{-/-}$infected pregnant mice were evidently smaller and shapeless, placentas were obvious more hemorrhagic and resorbed (Figure 1B). The weight of fetus or placenta from LILRB4 $4^{-/-}$infection group was less than that of WT infection group respectively (Figures $\mathbf{1 C}, \mathbf{D}$ ). The abortion rate of LILRB4 ${ }^{-/-}$infected group was significantly increased than that of WT infection group (Figure 1E). Thus, the knockout of LILRB4 aggravate the abnormal pregnancy outcome caused by T. gondii infection.

\section{The Dynamic LILRB4 Expression on Murine uDCs in Normal Pregnancy}

CD11c ${ }^{+}$cells were gated as uDCs shown in Figures 2A,B. LILRB4 expression on uDCs of normal pregnant mice at Gd 5, $8,10,12,14,16,18$, and non-pregnant mice was detected using flow cytometry (Figure 2C). The results showed that LILRB4 expression was at a lower level on uDCs from non-pregnant mice compared with the normal pregnant mice at all the designed time points. During pregnancy, LILRB4 expression gradually increased from Gd 5 to Gd 14, peaked on Gd 14, and decreased after Gd 14.
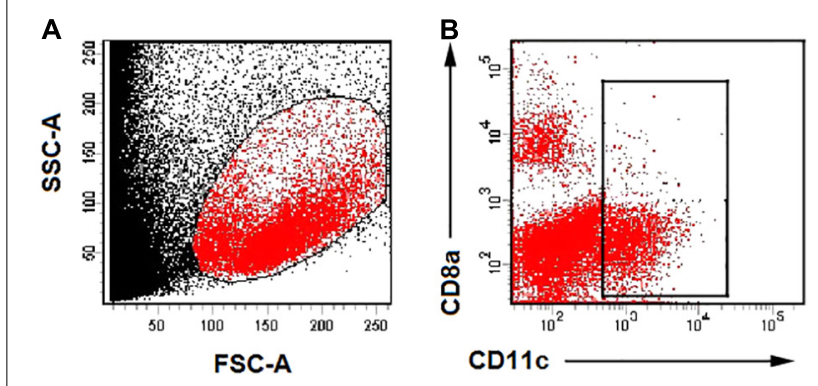

C

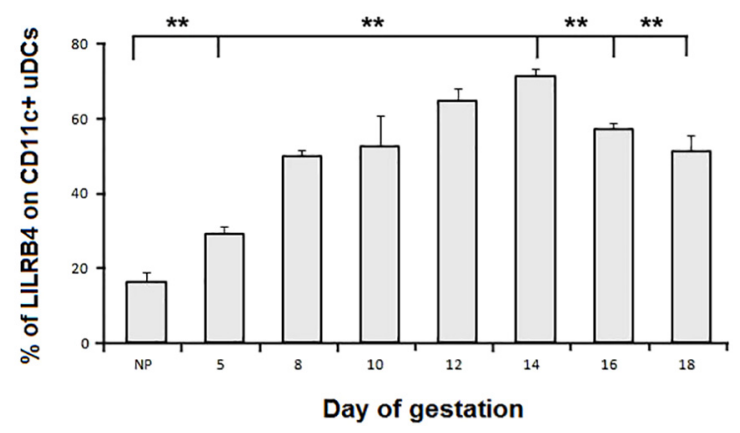

FIGURE 2 | Dynamic expression of LILRB4 on uterine DCs. (A) Decidual lymphocytes were gated in the appropriate location for flow cytometric analyses. (B) Uterine DCs were gated by CD11c expression. (C) Flow cytometry analyses of LILRB4 dynamic expression on CD11 $\mathrm{C}^{+}$uterine DCs were performed using non-pregnant mice and normal pregnant mice at day of gestation (Gd) 5, 8, 10, 12, 14, 16, 18, respectively. Data are shown as means $\pm \mathrm{SD}$ for 10 pregnant mice and differences were identified using unpaired $t$-tests $\left({ }^{*} p<0.05,{ }^{* *} p<0.01\right)$.

\section{LILRB4 Expression on uDCs Subsets in Both Mice and Human Was Down-Regulated After T. gondii Infection}

Flow cytometry analyses showed that LILRB4 was expressed on all the human MDC1, MDC2, and PDC subsets at a high level in normal pregnancy (Figures 3A,B). After T. gondii infection, the expression level of LILRB4 was significantly down-regulated on MDC2 and PDC subsets compared with the uninfected group. In normal pregnant mice, LILRB4 was expressed both on $\mathrm{CD} 11 \mathrm{c}^{+} \mathrm{CD} 8 \mathrm{a}^{-}$and $\mathrm{CD} 11 \mathrm{c}^{+} \mathrm{CD} 8 \mathrm{a}^{+} \mathrm{uDC}$ subsets. More importantly, LILRB4 expression on the tolerogenic CD11 $c^{+} \mathrm{CD}^{-} \mathrm{a}^{-} \mathrm{uDC}$ subset was much higher than that on the CD11 $\mathrm{c}^{+} \mathrm{CD} 8 \mathrm{a}^{+}$uDC subset (Figures 3C,D). After T. gondii infection, LILRB4 level decreased on the $\mathrm{CD} 11 \mathrm{c}^{+} \mathrm{CD} 8 \mathrm{a}^{-} \mathrm{uDC}$ subset (Figure 3E) while increased on $\mathrm{CD}_{11 \mathrm{c}^{+} \mathrm{CD} 8 \mathrm{a}^{+}} \mathrm{uDC}$ subset (Figure 3F).

\section{Expression of Functional Molecules on uDCs Was Changed When LILRB4 Is Down-Regulated by T. gondii Infection}

The expression levels of functional molecules CD80, CD86, and HLA-DR on human uDCs subsets were detected (Figure 4). After T. gondii infection, the expression of CD80, CD86, and HLA-DR were all up-regulated in the three human subsets 

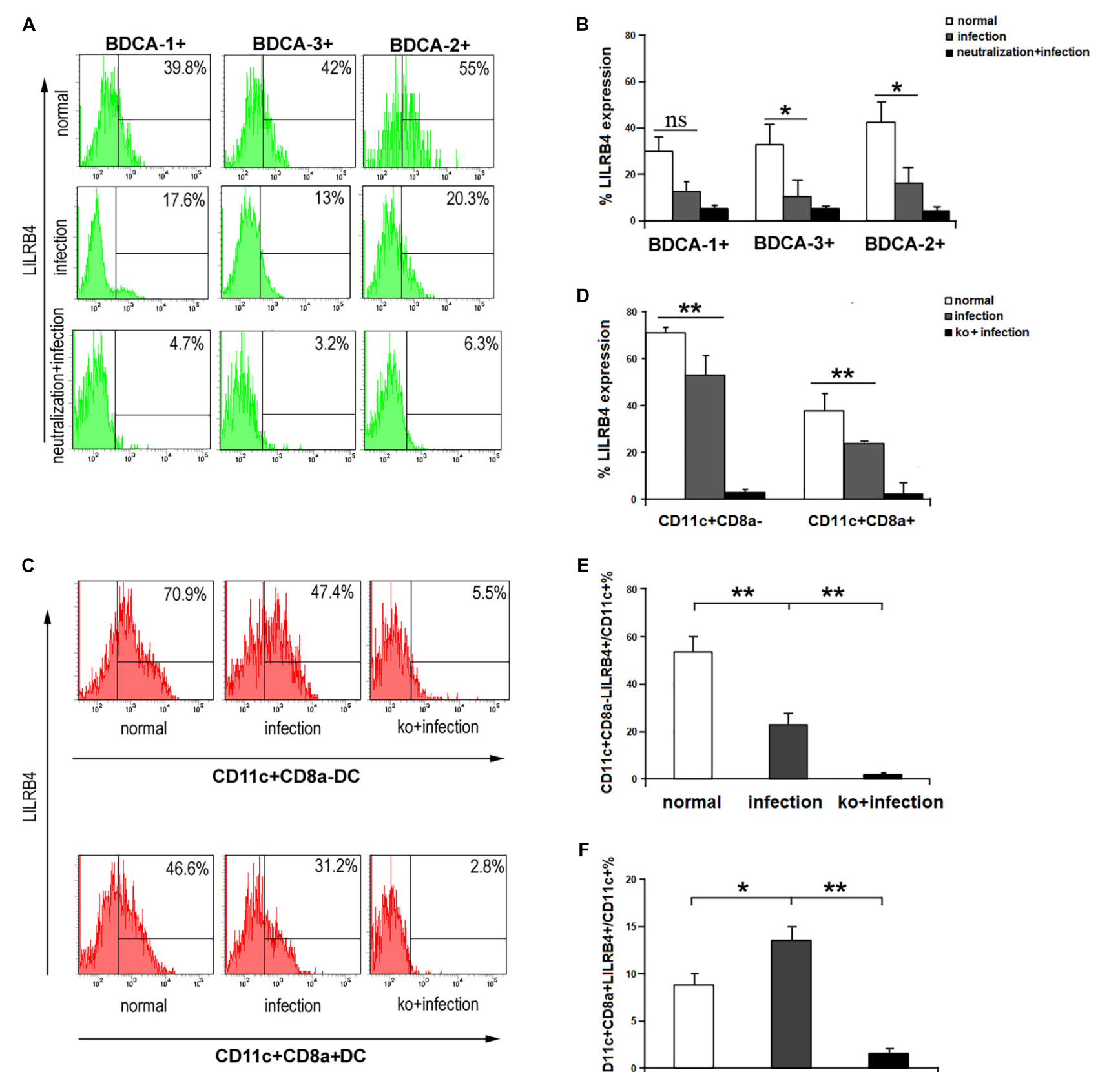

E
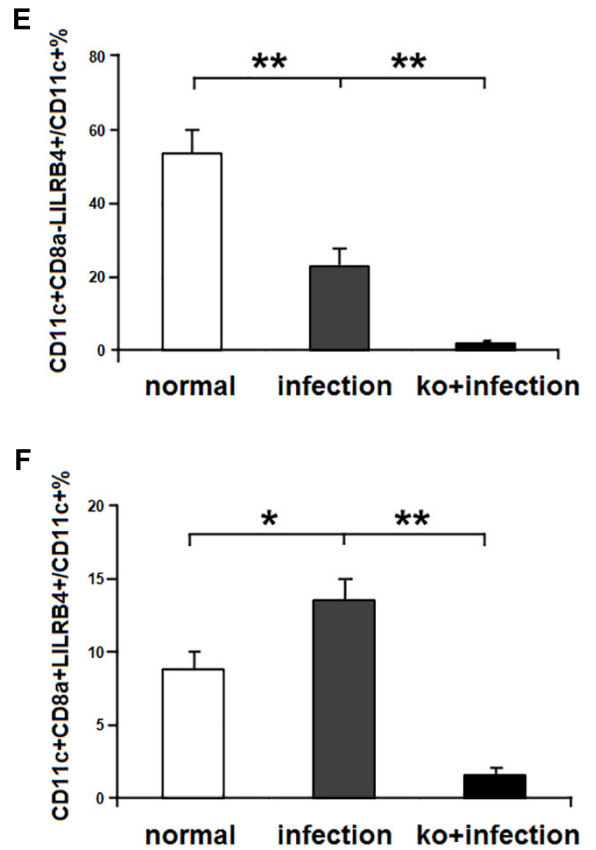

FIGURE 3 | Expression of LILRB4 on uDC subsets was down-regulated following T. gondii infection. (A) Flow cytometry analyses of LILRB4 expression on BDCA $-1^{+}(B D C A-1), B D C A-2^{+}$(BDCA-2), and BDCA-3 ${ }^{+}(\mathrm{PDC})$ UDC subsets were performed in normal, infected, and LILRB4-neutralized infected human uDCs. (B) LILRB4 expression changes were compared after $T$. gondii infection in human BDCA-1+, BDCA- $2^{+}$, and BDCA-3 ${ }^{+}$uDC subsets from normal, infected, and

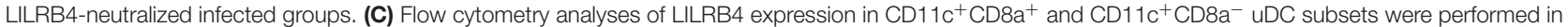

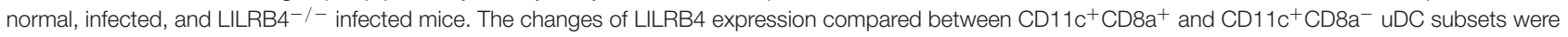

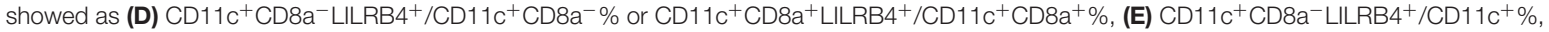

(F) CD11C ${ }^{+}$CD8a ${ }^{+}$LILRB $^{+} / \mathrm{CD} 11 \mathrm{C}^{+} \%$. Data are shown as means $\pm \mathrm{SD}\left({ }^{*} p<0.05,{ }^{* *} p<0.01\right)$. Representative data from 10 independent experiments for each group.

compared with the uninfected group. On uDCs which LILRB4 was neutralized, CD80, CD86, and HLA-DR were further up-regulated compared with the infected cells. The in vivo studies showed that the levels of CD80, CD86, and MHC II on tolerogenic $\mathrm{CD} 11 \mathrm{c}^{+} \mathrm{CD} 8 \alpha^{-}$uDC subset were significantly lower than on $\mathrm{CD} 11 \mathrm{c}^{+} \mathrm{CD} 8 \alpha^{+} \mathrm{uDC}$ subset during normal mice pregnancy (Figure 5). After T. gondii infection, CD80, CD86, and $\mathrm{MHC}$ II on murine $\mathrm{CD} 11 \mathrm{c}^{+} \mathrm{CD} 8 \alpha^{-}$and $\mathrm{CD} 11 \mathrm{c}^{+} \mathrm{CD} 8 \alpha^{+}$ 

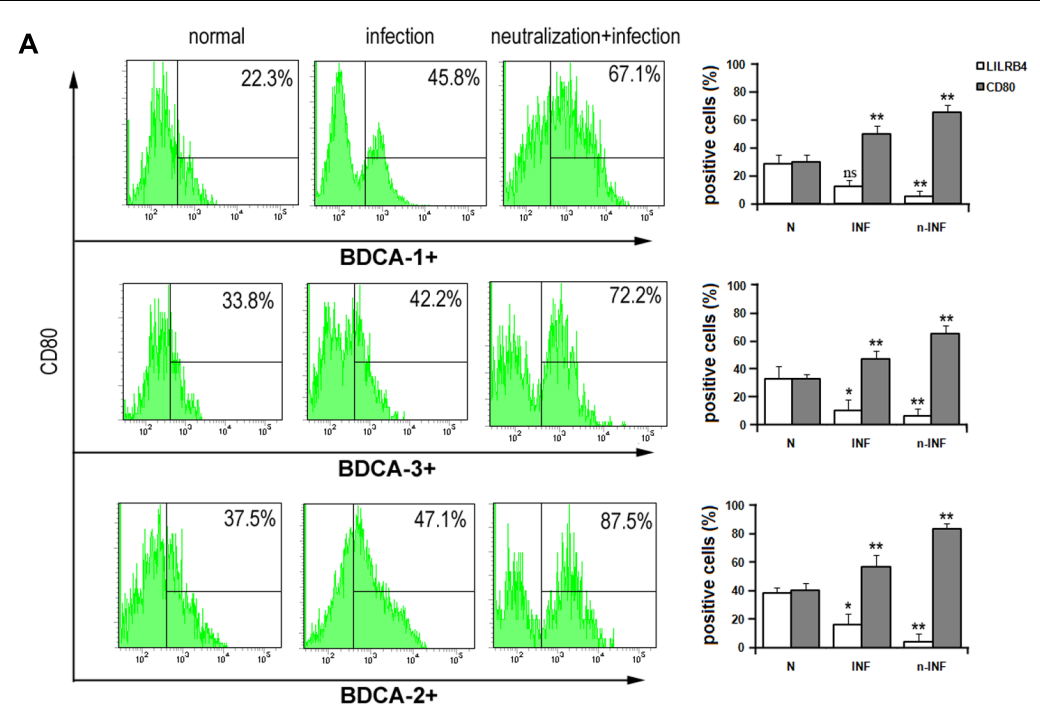

B
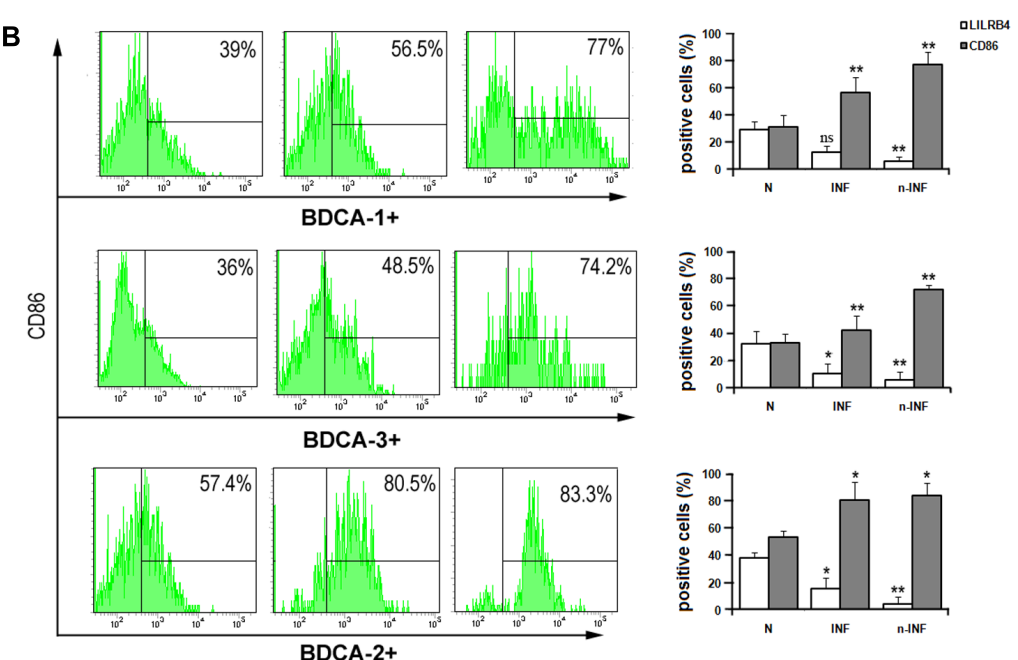

C
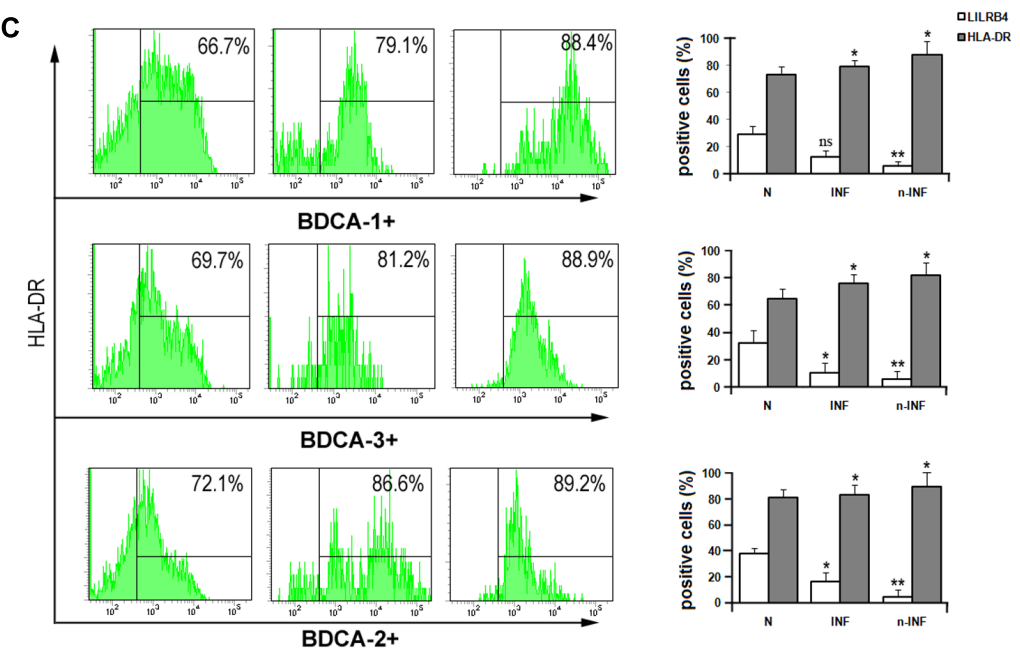

FIGURE 4 | Down-regulation of LILRB4 on human UDC subsets with T. gondii infection resulted in changes in the expression of functional molecules of human uDCs. Flow cytometry and histogram analyses of CD80 (A), CD86 (B), HLA-DR (C) and LILRB4 expression in BDCA-1+ , BDCA-2+ , and BDCA-3+ uDC subsets were performed for normal, infected, and anti-LILRB4 neutralizing antibody-treated infected human uDCs. Data are shown as means $\pm \mathrm{SD}\left({ }^{*} p<0.05,{ }^{* *} p<0.01\right)$. Representative data from six independent experiments for each group. 


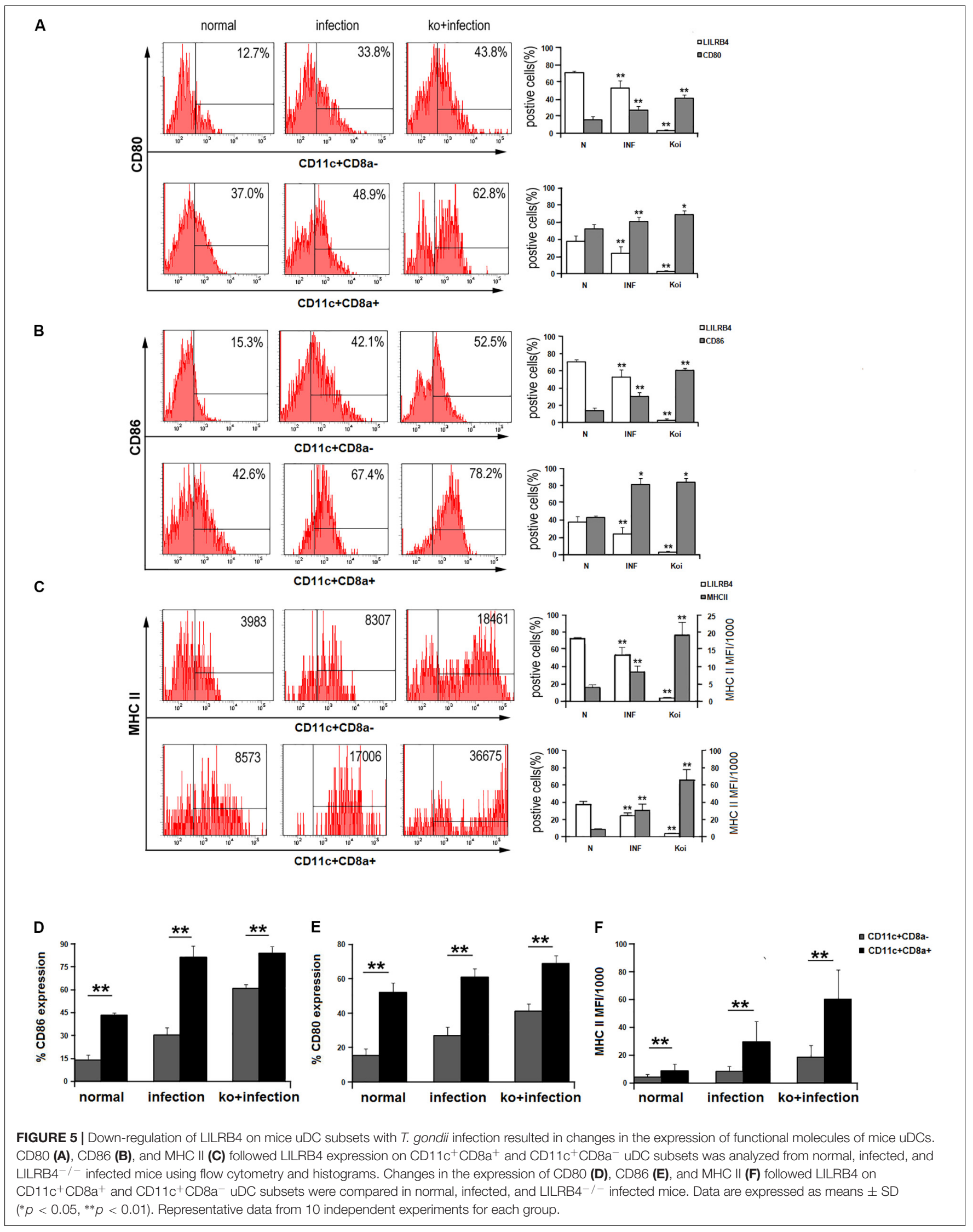


uDC subsets were both up-regulated, and they were further increased in LILRB4 ${ }^{-/-}$infected mice compared with the infected mice (Figures 5A-C). And the up-regulation of these functional molecules was more obvious on the tolerogenic $\mathrm{CD} 11 \mathrm{c}^{+} \mathrm{CD} 8 \alpha^{-} \mathrm{uDC}$ subset than $\mathrm{CD} 11 \mathrm{c}^{+} \mathrm{CD} 8 \alpha^{+} \mathrm{uDC}$ subset (Figures 5D-F).

\section{DISCUSSION}

It was reported that the tolerogenic functions of DCs are characterized by high expression of the inhibitory receptor LILRB4 and low co-stimulatory potential (Chang et al., 2002). During normal pregnancy, LILRB4 can modulate the functions of APCs and plays important roles in immune regulation and tolerance at the maternal-fetal interface (Matsumoto et al., 2001). Although previous studies have shown that LILRB4 is expressed on uDCs during normal pregnancy, the dynamic expression levels remain unclear. In the present study, the level of LILRB4 expression throughout murine gestation was measured by flow cytometry. The results showed that LILRB4 expression was at the lower level in non-pregnant mice uDCs, gradually increased from the first trimester to the middle-late trimester, and peaking on day 14 of gestation in mice. More importantly, LILRB4 expression levels on tolerogenic mice $\mathrm{CD} 11 \mathrm{c}^{+} \mathrm{CD} 8 \mathrm{a}^{-}$ $\mathrm{uDC}$ subsets were higher than that on other subsets during normal pregnancy. These data suggest that LILRB4 on uDCs, especially tolerogenic uDC subsets, may be beneficial for normal pregnancy.

Our previous study showed that more severely adverse pregnancy outcomes was observed in $T$. gondii-infected LILRB4 $^{-/-}$pregnant mice, that indicated LILRB4 was related to the development of abnormal pregnancy outcomes following $T$. gondii infection and have found that the expression of LILRB4 on decidual macrophage was detected downregulated ( $\mathrm{Li}$ et al., 2017). Whether T. gondii infection could affect LILRB4 expression on uDCs and whether this effect subsequently contributes to abnormal pregnancy outcomes were all still unclear. In present study, in order to further investigate whether $T$. gondii infections could affect LILRB4 expression on uDCs subsets, LILRB4 expression was monitored on uDCs during $T$. gondii infection both in vitro and in vivo. The results showed LILRB4 expression to be significantly down-regulated by $T$. gondii infection on both human and mouse uDCs during pregnancy, and LILRB4 down-regulation were mainly on tolerogenic murine $\mathrm{CD} 11 \mathrm{c}^{+} \mathrm{CD}^{-} \mathrm{a}^{-} \mathrm{uDC}$ subsets rather than on other uDC subsets. Hence, the results suggest that LILRB4 downregulation on uDCs, especially tolerogenic uDC subsets, may play a role in the development of $T$. gondii-mediated abnormalities.

To further assess a mechanistic basis for these observations, membrane functional molecules CD80, CD86, and HLA-DR (MHC II in mice) on uDCs were examined during T. gondii infection. The functions of DCs are characterized partly by dynamic regulation of co-stimulatory molecules (CD80, CD86) and HLA-DR (McLellan et al., 1995; Nijman et al., 1995).
The human decidual MDC1 (BDCA-1 ${ }^{+}$) subset expresses low levels of CD80, CD86, and HLA-DR and is regarded as an immature decidual MDC subset involved in inducing immune tolerance (Gardner and Moffett, 2003; Mahnke et al., 2003). MDC2 $\left(\mathrm{BDCA}-3^{+}\right)$is similar to MDC1 with respect to the phenotype. LILRB4 ${ }^{\text {high }}$ MDC2, which has a reduced allostimulatory capacity is considered a feature of tolerogenic DCs (Velten et al., 2004). PDC (BDCA-2 ${ }^{+}$), which has a lower level of CD80 and CD86, was reported to contribute to the maintenance of normal pregnancy at the maternal-fetal interface (Miyazaki et al., 2003). So, the three subsets of u DC all are important for the normal human pregnancy. In the present study, the results showed that CD80, CD86, and HLA-DR in human MDC1, MDC2, and PDC were significantly upregulated after $T$. gondii infection followed LILRB4 decrease. These data suggested that $T$. gondii infection could significantly weaken the immune tolerogenic function in uDCs by downregulating LILRB4 expression and enhance immune-activated functions by up-regulating functional molecules CD80, CD86, and HLA-DR expression. To further clarify the role of LILRB4 in the adverse pregnancy outcome caused by $T$. gongdii infection, we performed experiments in which LILRB4 was neutralized with antibody in vitro to assess CD80, CD86, and HLA-DR expression. The results showed that functional molecules CD80, CD86, and HLA-DR expression in LILRB4neutralized infected human MDC1, MDC2, and PDC subsets were further up-regulated compared with the infected uDC subsets. The in vivo study showed that, followed the decrease of LILRB4, T. gondii infection significantly induced functional molecules CD80, CD86, and MHC II expressions on mice uDCs subsets, and more importantly, the up-regulation of functional molecules were more obvious on tolerogenic $\mathrm{CD} 11 \mathrm{c}^{+} \mathrm{CD} 8 \mathrm{a}^{-}$ $\mathrm{uDC}$ subset than $\mathrm{CD} 11 \mathrm{c}^{+} \mathrm{CD} 8 \mathrm{a}^{+} \mathrm{uDC}$ subset. The results of T. gondii-infected LILRB4 ${ }^{-/-}$pregnant mice that the functional molecules (CD80, CD86, and MHC II) on the two uDC subsets were both further up-regulated compared with the infected WT mice and more obviously on the tolerogenic $\mathrm{CD} 11 \mathrm{c}^{+} \mathrm{CD} 8 \mathrm{a}^{-} \mathrm{uDC}$ subset, further confirmed the changes of the functional molecules resulted from LILRB4 down-regulation after $T$. gondii infection. The results demonstrated that the changes in functional molecules (CD80, CD86, and MHC II) are associated with decreased LILRB4 expression on uDCs, especially in tolerogenic uDC subsets, during $T$. gondii infection. A disturbance of uDC tolerance function, due to change in LILRB4 and functional molecules, may contribute to the development of abnormal pregnancy outcomes during T. gondii infection.

\section{CONCLUSION}

The results of this study show that down-regulation of LILRB4 on uDCs, especially on tolerogenic uDC subset, following T. gondii infection weakened immune-tolerogenic function of $\mathrm{uDC}$ by up-regulating functional molecules CD80, CD86, and HLA-DR (MHC II) expression and ultimately contributed to abnormal pregnancy outcomes by $T$. gondii infection. This investigation 
further shed light on the molecular immune mechanisms of uDCs in abnormal pregnancy outcomes due to T. gondii infection.

\section{AUTHOR CONTRIBUTIONS}

SZ, JZ, HZ, and XH designed the study and edited the manuscript. SZ, JZ, HZ, and LR performed the mouse experiments. XL, JZ, YJ, CY, LR, and MZ provided the samples and performed the patients experiments. SZ, HZ, and XH wrote the manuscript. SZ, JZ, and HZ made equal contributions to this

\section{REFERENCES}

Adorini, L., Giarratana, N., and Penna, G. (2004). Pharmacological induction of tolerogenic dendritic cells and regulatory T cells. Semin. Immunol. 16, 127-134. doi: 10.1016/j.smim.2003.12.008

Ban, Y. L., Kong, B. H., Qu, X., Yang, Q. F., and Ma, Y. Y. (2008). BDCA-1, BDCA-2 and BDCA-3 dendritic cells in early human pregnancy decidua. Clin. Exp. Immunol. 151, 399-406. doi: 10.1111/j.1365-2249.2007. 03576.x

Blois, S. M., Alba Soto, C. D., Tometten, M., Klapp, B. F., Margni, R. A., and Arck, P. C. (2004). Lineage, maturity, and phenotype of uterine murine dendritic cells throughout gestation indicate a protective role in maintaining pregnancy. Biol. Reprod. 70, 1018-1023. doi: 10.1095/biolreprod.103.022640

Cella, M., Döhring, C., Samaridis, J., Dessing, M., Brockhaus, M., and Lanzavecchia, A. (1997). A novel inhibitory receptor (ILT3) expressed on monocytes, macrophages, and dendritic cells involved in antigen processing. J. Exp. Med. 185, 1743-1751. doi: 10.1084/jem.185.10. 1743

Chang, C. C., Ciubotariu, R., Manavalan, J. S., Yuan, J., Colovai, A. I., Piazza, F., et al. (2002). Tolerization of dendritic cells by TS cells: the crucial role of inhibitory receptors ILT3 and ILT4. Nat. Immunol. 3, 237-243. doi: 10.1038/ ni760

Chang, C. C., Liu, Z., Vlad, G., Qin, H., Qiao, X., Mancini, D. M., et al. (2009). Ig-like transcript 3 regulates expression of proin-flammatory cytokines and migration of activated T cells. J. Immunol. 182, 5208-5216. doi: 10.4049/ jimmunol.0804048

Della Bella, S., Giannelli, S., Cozzi, V., Signorelli, V., Cappelletti, M., Cetin, I., et al. (2011). Incomplete activation of peripheral blood dendritic cells during healthy human pregnancy. Clin. Exp. Immunol. 164, 180-192. doi: 10.1111/j.1365-2249. 2011.04330.x

Gardner, L., and Moffett, A. (2003). Dendritic cells in the human decidua. Biol. Reprod. 69, 1438-1446. doi: 10.1095/biolreprod.103.01 7574

Guleria, I., and Sayegh, M. H. (2007). Maternal acceptance of the fetus: true human tolerance. J. Immunol. 178, 3345-3351. doi: 10.4049/jimmunol.178.6. 3345

Hunt, J. S., and Robertson, S. A. (1996). Uterine macrophages and environmental programming for pregnancy success. J. Reprod. Immunol. 32, 1-25. doi: 10.1016/S0165-0378(96)88352-5

Kasai, S., Inui, M., Nakamura, K., Kakizaki, Y., Endo, S., Nakamura, A., et al. (2008). A novel regulatory role of gp49B on dendritic cells in T-cell priming. Eur. J. Immunol. 38, 2426-2437. doi: 10.1002/eji.20073 7550

Kim-Schulze, S., Seki, T., Vlad, G., Scotto, L., Fan, J., Colombo, P. C., et al. (2006). Regulation of ILT3 gene expression by processing of precursor transcripts in human endothelial cells. Am. J. Transplant. 6, 76-82. doi: 10.1111/j.1600-6143. 2005.01162.x

Langenkamp, A., Messi, M., Lanzavecchia, A., and Sallusto, F. (2000). Kinetics of dendritic cell activation: impact on priming of TH1, TH2 and nonpolarized $\mathrm{T}$ cells. Nat. Immunol. 1, 311-316. doi: 10.1038/ 79758

Laskarin, G., Kämmerer, U., Rukavina, D., Thomson, A. W., Fernandez, N., and Blois, S. M. (2007). Antigen-presenting cells and materno-fetal tolerance: paper. All authors read the final version of the manuscript and approved it for publication.

\section{FUNDING}

This work was supported by funds from the National Natural Science Foundation of China (Grant Nos. 81672049, 81401687), the Natural Science Foundation of Shandong Province (Grant No. ZR2013HQ023), and the Taishan Scholar Foundation of Shandong Province.

an emerging role for dendritic cells. Am. J. Reprod. Immunol. 58, 255-267. doi: 10.1111/j.1600-0897.2007.00511.x

Li, Z., Zhao, M., Li, T., Zheng, J., Liu, X., Jiang, Y., et al. (2017). Decidual macrophage functional polarization during abnormal pregnancy due to Toxoplasma gondii: role for LILRB4. Front. Immunol. 8:1013. doi: 10.3389/ fimmu.2017.01013

Liu, X., Zhao, M., Yang, X., Han, M., Xu, X., Jiang, Y., et al. (2014). Toxoplasma gondii infection of decidual $\mathrm{CD} 1 \mathrm{c}(+)$ dendritic cells enhances cytotoxicity of decidual natural killer cells. Inflammation 37, 1261-1270. doi: 10.1007/s10753014-9853-X

Liu, Y., Zhao, M., Xu, X., Liu, X., Zhang, H., Jiang, Y., et al. (2014). Adoptive transfer of Treg cells counters adverse effects of Toxoplasma gondii infection on pregnancy. J. Infect. Dis. 210, 1435-1443. doi: 10.1093/infdis/ jiu265

Mahnke, K., Qian, Y., Knop, J., and Enk, A. H. (2003). Induction of CD4+/CD25+ regulatory $\mathrm{T}$ cells by targeting of antigens to immature dendritic cells. Blood 101, 4862-4869. doi: 10.1182/blood-2002-10-3229

Matsumoto, Y., Handa, S., and Taki, T. (1997). gp49B1, an inhibitory signaling receptor gene of hematopoietic cells, is induced by leukemia inhibitory factor in the uterine endometrium just before implantation. Dev. Growth Differ. 39, 591-597. doi: 10.1046/j.1440-169X.1997.t01-400006.x

Matsumoto, Y., Wang, L. L., Yokoyama, W. M., and Aso, T. (2001). Uterine macrophages express the gp49B inhibitory receptor in midgestation. J. Immunol. 166, 781-786. doi: 10.4049/jimmunol.166. 2.781

McLellan, A. D., Starling, G. C., Williams, L. A., Hock, B. D., and Hart, D. N. (1995). Activation of human peripheral blood dendritic cells induces the CD86 co-stimulatory molecule. Eur. J. Immunol. 25, 2064-2068. doi: 10.1002/eji. 1830250739

Miyazaki, S., Tsuda, H., Sakai, M., Hori, S., Sasaki, Y., Futatani, T., et al. (2003). Predominance of Th2-promoting dendritic cells in early human pregnancy decidua. J. Leukoc. Biol. 74, 514-522. doi: 10.1189/jlb.110 2566

Nijman, H. W., Kleijmeer, M. J., Ossevoort, M. A., Oorschot, V. M., Vierboom, M. P., and van de Keur, M. (1995). Antigen capture and major histocompatibility class II compartments of freshly isolated and cultured human blood dendritic cells. J. Exp. Med. 182, 163-174. doi: 10.1084/jem.182. 1.163

Regnault, A., Lankar, D., Lacabanne, V., Rodriguez, A., Thery, C., and Rescigno, M. (1999). Fcgamma receptor-mediated induction of dendritic cell maturation and major histocompatibility complex class I-restricted antigen presentation after immune complex internalization. J. Exp. Med. 189, 371-380. doi: 10.1084/jem. 189.2.371

Robbins, J. R., Zeldovich, V. B., Poukchanski, A., Boothroyd, J. C., and Bakardjiev, A. I. (2012). Tissue barriers of the human placenta to infection with Toxoplasma gondii. Infect. Immun. 80, 418-428. doi: 10.1128/IAI.058 99-11

Velten, F. W., Duperrier, K., Bohlender, J., Metharom, P., and Goerdt, S. (2004). A gene signature of inhibitory MHC receptors identifies a BDCA3(+) subset of IL-10-induced dendritic cells with reduced allostimulatory capacity in vitro. Eur. J. Immunol. 34, 2800-2811. doi: 10.1002/eji.20032 4732 
Vlad, G., Chang, C. C., Colovai, A. I., Vasilescu, E. R., Cortesini, R., and SuciuFoca, N. (2010). Membrane and soluble ILT3 are critical to the generation of T suppressor cells and induction of immunological tolerance. Int. Rev. Immunol. 29, 119-132. doi: 10.3109/08830180903281185

Xu, X., Fu, Q., Zhang, Q., Zhao, M., Gao, Z., Liu, X., et al. (2013). Changes of human decidual natural killer cells cocultured with YFP-Toxoplasma gondii: implications for abnormal pregnancy. Fertil. Steril. 99, 427-432. doi: 10.1016/ j.fertnstert.2012.09.016

Zhao, M., Zhang, R., Xu, X., Liu, Y., Zhang, H., Zhai, X., et al. (2013). IL10 reduces levels of apoptosis in Toxoplasma gondii-infected trophoblasts. PLoS One 8:e56455. doi: 10.1371/journal.pone.0056455
Conflict of Interest Statement: The authors declare that the research was conducted in the absence of any commercial or financial relationships that could be construed as a potential conflict of interest.

Copyright $\odot 2018$ Zhan, Zheng, Zhang, Zhao, Liu, Jiang, Yang, Ren, Liu and Hu. This is an open-access article distributed under the terms of the Creative Commons Attribution License (CC BY). The use, distribution or reproduction in other forums is permitted, provided the original author(s) and the copyright owner are credited and that the original publication in this journal is cited, in accordance with accepted academic practice. No use, distribution or reproduction is permitted which does not comply with these terms. 